\title{
Entanglement of a two-atom system driven by the quantum vacuum in arbitrary cavity size
}

\author{
G. Flores-Hidalgd* \\ Instituto de Física e Química, Universidade Federal de Itajubá, 37500-903, Itajubá, MG, Brazil. \\ M. Rojast and Onofre Rojast \\ Departamento de Física, Universidade Federal de Lavras, CP 3037, 37200-000, Lavras, MG, Brazil.
}

\begin{abstract}
We study the dynamical entanglement of two identical atoms interacting with a quantum field. As a simplified model for this physical system we consider two harmonic oscillators linearly coupled to a massless scalar field in the dressed coordinates and states approach and enclose the whole system inside a spherical cavity of radius $R$. Through a quantity called concurrence, the entanglement evolution for the two-atom system will be discussed, for a range of initial states composed of a superposition of atomic states. Our results reveals how the concurrence of the two atoms behaves through the time evolution, for arbitrary cavity size and for arbitrary coupling constant, weak, intermediate or strong. All our computations are exact and only the final step is numerical. These numerical solutions give us fascinating results for the concurrence, such as quasi-random fluctuations, with a resemblance of periodicity. Another interesting result we found is when the system is initially maximally entangled (disentangled), after the time $t=2 R$, the system becomes again strongly entangled (disentangled) particularly during the first oscillations, later this phenomenon could be wrecked depending on the initial condition. We also show the concurrence after a too long time elapsed with a good precision.
\end{abstract}

PACS: 03.67.Mn, 03.67.Bg

\section{INTRODUCTION}

Entanglement is one of the most exciting topics in quantum mechanics, due it's unusual property of nonlocality, which has been considering as a key source in quantum information processing. During the past decade, due the rapid development of the experimental process in quantum control, there has been a rapidly growing interest in entanglement generation. There are potential applications in nearly all quantum communication and computation protocols [1], such as quantum teleportation [2, 3], quantum secure direct communication [4], quantum computation [5, 6] among others.

One of the simplest schemes in which entanglement can be generated is a system containing a couple of two-level atoms. In 90 decade, two-atom entangled states have already been demonstrated experimentally using ultra cold trap ions [7, 8] and cavity quantum electrodynamics (QED) schemes [9]. It has been showed that entangled states can be generated in a two-atom system by a continuous driving of the atoms with a coherent or chaotic thermal field [10, 11], or by a pulse excitation followed by a continuous radiative decay $12-14]$. In order to confront the experimental results, several theoretical studies have been considered for the preparation of a two-atom system in an entangled state. In references [16, 17] the entanglement of two atoms by spontaneous emission for a model that includes dipole-dipole interaction between

\footnotetext{
* gfloreshidalgo@unifei.edu.br

† moises.leyva@dfi.ufla.br

$\ddagger$ ors@dfi.ufla.br
}

the atoms have been discussed. The authors studied both numerically and analytically the concurrence and negativity for such a system. Also, in reference [18] the authors discussed numerical solutions for the concurrence in a system of two atoms driven by a laser field.

In this work, we study the entanglement of a two-atom system driven by the quantum vacuum field, the whole system enclosed inside a spherical cavity of radius $R$. As simplified model of the atoms we consider each one as single harmonic oscillator and the radiation field is taken as a massless scalar field. In order to give physical sense to our model we will work in the dressed coordinates and dressed states approach, that were introduced some time ago in references [19 21], in analogy with the renormalized fields in quantum field theory. Such dressed coordinates and states have been defined in the physical context of an atom, approximated by an harmonic oscillator, linearly coupled to a scalar field and confined in a spherical cavity of radius $R$. In terms of dressed coordinates, dressed states have been defined as the physically measurable states. The dressed states having the physical correct property of stability of the oscillator (atom) ground state in the absence of field quanta (the quantum vacuum). For a clear explanation see reference [25]. Also, the formalism showed to have the technical advantage of allowing exact computation of the probabilities associated with the different oscillator (atom) radiation processes [26]. For example, it has been obtained easily the probability of the atom to decay spontaneously from the first excited state to the ground state for arbitrary coupling constant, weak or strong and for arbitrary cavity size. For weak coupling constant in the limit $R \rightarrow \infty$, that corresponds to free space, the old know result: $e^{-\Gamma t}$, has been obtained [19]. Also, considering 
a cavity of sufficiently small radius [20], the method accounted for the experimentally observed inhibition of the spontaneous decaying processes of the atom [22, 23]. In references [24, 25] the concept of dressed coordinates and states have been extended to the case in which nonlinear interactions between the oscillator and the field modes are taken into account. Furthermore, in [27] the oscillator electromagnetic field interaction has been considered and in reference 28] dressed coordinates and states have been introduced in the path integral formalism.

This work is organized as follow. In section II we describe the model of the two atoms coupled to a massless scalar field, find the eigenvalues and eigenfunctions of the system and define the dressed coordinates and states. In section III we compute some probability amplitudes, that will be useful for further study of the entanglement of the two-atom system inside the spherical cavity. In section IV we discuss the entanglement of the two-atom system through the quantitity called of concurrence, illustrating the behaviour of this quantity as a funtion of time, coupling constant and cavity radius. Finally, in section $\mathrm{V}$ we give our conclusions. We consider natural units $\hbar=c=1$.

\section{THE MODEL}

Let us consider two identical atoms inside a cavity linearly coupled to a massless scalar field. Roughly approximating the atoms by two harmonic oscillators of frequency $\omega_{0}$, the system can be described by the following Hamiltonian,

$$
\begin{aligned}
H= & \frac{1}{2}\left(p_{A}^{2}+\omega_{0}^{2} q_{A}^{2}\right)+\frac{1}{2}\left(p_{B}^{2}+\omega_{0}^{2} q_{B}^{2}\right)+\frac{1}{2} \sum_{k=1}^{N}\left(p_{k}^{2}+\omega_{k}^{2} q_{k}^{2}\right)+ \\
& +\frac{1}{\sqrt{2}} \sum_{k=1}^{N} c_{k} q_{k}\left(q_{A}+q_{B}\right)+\sum_{k=1}^{N} \frac{c_{k}^{2}}{4 \omega_{k}}\left(q_{A}+q_{B}\right)^{2},
\end{aligned}
$$

where the coordinates and momenta $q_{A}, p_{A}$ and $q_{B}, p_{B}$ correspond to the atoms $A$ and $B$ respectively, $\omega_{k}$ is the frequency of the field modes and $c_{k}$ the coupling constant of the atoms with the scalar field. The coordinates and momenta $q_{k}$ and $p_{k}$ are related to the field modes of frequencies $\omega_{k}$. The last term in Eq. (10) is introduced to guarantee the positiveness of the Hamiltonian and can be understood as a renormalization frequency for the particles oscillators $[30$. At the end of the calculation, we will take $N \rightarrow \infty$. Also $\omega_{k}=\pi k / R, k=\{0,1,2, \ldots\}$, where $R$, is the radius in which the whole system is confined. Surely, we can recover the free space case taking the limit $R \rightarrow \infty$.

The model Hamiltonian given by Eq. (10) has been introduced in Ref. [31] and used to study the entanglement between two single harmonics oscillators in Ref. [32]. Here we re-derive above Hamiltonian by considering two identical harmonic oscillator located at positions $\mathbf{r}_{A}$ and $\mathbf{r}_{B}$, interacting with a massless scalar field, the whole system inside a spherical cavity or radius $R$. The Lagrangian of the system is

$$
\begin{aligned}
L= & \frac{1}{2}\left(\dot{q}_{A}^{2}-\omega_{0}^{2} q_{A}^{2}\right)+\frac{1}{2}\left(\dot{q}_{B}^{2}-\omega_{0}^{2} q_{B}^{2}\right)+ \\
& +\frac{1}{2} \int d^{3} \mathbf{r}\left(\left(\frac{\partial \phi}{\partial t}\right)^{2}-(\nabla \phi)^{2}\right)+ \\
& -\sqrt{g} \int d^{3} \mathbf{r} \phi(\mathbf{r}, t)\left(\delta\left(\mathbf{r}-\mathbf{r}_{A}\right) q_{A}+\delta\left(\mathbf{r}-\mathbf{r}_{B}\right) q_{B}\right),
\end{aligned}
$$

where the coupling constant is written in such a way that $g$ has dimension of frequency. Note that the two harmonic oscillators oscillate in another abstract space than spatial $\Re^{3}$. The coordinates $q_{A}$ and $q_{B}$ must be viewed as coordinates representing all the relevant internal degrees of freedom of the subsystems $A$ and $B$. We are also considering unit mass for the particle oscillators. For other masses the Lagrangian can be transformed into Eq. (2), through a scale coordinate transformation. Now, suppose that the particle oscillators (atoms) are located nearly at the center of the cavity, this means that $\mathbf{r}_{A} \approx \mathbf{r}_{B} \approx 0$. Therefore, the Lagrangian becomes

$$
\begin{aligned}
L= & \frac{1}{2}\left(\dot{q}_{A}^{2}-\omega_{0}^{2} q_{A}^{2}\right)+\frac{1}{2}\left(\dot{q}_{B}^{2}-\omega_{0}^{2} q_{B}^{2}\right)+ \\
& +\frac{1}{2} \int d^{3} \mathbf{r}\left(\left(\frac{\partial \phi}{\partial t}\right)^{2}-(\nabla \phi)^{2}\right)+ \\
& -\sqrt{g} \phi(\mathbf{0}, t)\left(q_{A}+q_{B}\right) .
\end{aligned}
$$

Expanding the field $\phi(\mathbf{r}, t)$ as

$$
\phi(\mathbf{r}, t)=\sum_{k} q_{k}(t) u_{k}(\mathbf{r}),
$$

where $\left\{u_{k}(\mathbf{r})\right\}$ is a complete orthonormal set of solutions of the equation,

$$
-\nabla^{2} u_{k}(\mathbf{r})=\omega_{k}^{2} u_{k}(\mathbf{r}), \quad u_{k}(R)=0,
$$

where we are imposing Dirichlet boundary conditions for the field, $\phi(R, t)=0$. Replacing (4) in (3), using Eq. (5) and orthonormality property, we get

$$
\begin{aligned}
L= & \frac{1}{2}\left(\dot{q}_{A}^{2}-\omega_{0}^{2} q_{A}^{2}\right)+\frac{1}{2}\left(\dot{q}_{B}^{2}-\omega_{0}^{2} q_{B}^{2}\right)+\frac{1}{2} \sum_{k}\left(\dot{q}_{k}^{2}-\omega_{k}^{2} q_{k}^{2}\right) \\
& -\sqrt{g} \sum_{k} u_{k}(0) q_{k}\left(q_{A}+q_{B}\right) .
\end{aligned}
$$

From above equation we can note that the field modes interacting with the particle oscillators are such that $u_{k}(0) \neq 0$. Solving Eq. (5), it can be showed that such solutions are the spherically symmetric ones, i.e,

$$
u_{k}(\mathbf{r})=\frac{\sin \left(\omega_{k} r\right)}{r \sqrt{2 \pi R}}
$$

where

$$
\omega_{k}=\frac{\pi k}{R}, k=1,2,3, \ldots, N \rightarrow \infty
$$


Using Eq. (7), we get

$$
u_{k}(0)=\frac{\omega_{k}}{\sqrt{2 \pi R}} .
$$

Substituting Eq. (9) in Eq. (6), we obtain

$$
\begin{aligned}
L= & \frac{1}{2}\left(\dot{q}_{A}^{2}-\omega_{0}^{2} q_{A}^{2}\right)+\frac{1}{2}\left(\dot{q}_{B}^{2}-\omega_{0}^{2} q_{B}^{2}\right)+\frac{1}{2} \sum_{k}\left(\dot{q}_{k}^{2}-\omega_{k}^{2} q_{k}^{2}\right) \\
& -\sqrt{g} \sum_{k} u_{k}(0) q_{k}\left(q_{A}+q_{B}\right) .
\end{aligned}
$$

From which, it is not difficult to obtain the Hamiltonian (1), with $c_{k}$ given by

$$
c_{k}=\frac{\omega_{k}}{\pi \sqrt{2}} \sqrt{g \Delta \omega_{k}}, \quad \Delta \omega_{k}=\frac{\pi}{R} .
$$

In order to diagonalize the Hamiltonian (11), we introduce the relative and the center of mass coordinates $q_{-}$ and $q_{0}$, respectively and given by

$$
q_{-}=\frac{1}{\sqrt{2}}\left(q_{A}-q_{B}\right), \quad q_{0}=\frac{1}{\sqrt{2}}\left(q_{A}+q_{B}\right) .
$$

Substituting the above relations in Eq. (11), we have

$$
\begin{aligned}
H= & \frac{1}{2}\left(p_{-}^{2}+\omega_{0}^{2} q_{-}^{2}\right)+\frac{1}{2}\left(p_{0}^{2}+\omega_{0}^{2} q_{0}^{2}\right)+\frac{1}{2} \sum_{k=1}^{N}\left(p_{k}^{2}+\omega_{k}^{2} q_{k}^{2}\right)+ \\
& +\sum_{k=1}^{N} c_{k} q_{k} q_{0}+\sum_{k=1}^{N} \frac{c_{k}^{2}}{2 \omega_{k}} q_{0}^{2} .
\end{aligned}
$$

Note that in the Hamiltonian (13), the relative coordinate $q_{-}$is decoupled, thus, one can diagonalize the Hamiltonian ignoring the first term. For this purpose, we introduce, the collective coordinates and momenta, $Q_{r}$ and $P_{r}$, given by

$$
q_{\mu}=\sum_{r} t_{\mu}^{r} Q_{r}, \quad p_{\mu}=\sum_{r} t_{\mu}^{r} P_{r},
$$

where $\mu=0, k$, and $t_{\mu}^{r}$ is given by

$$
t_{k}^{r}=\frac{c_{k}}{\omega_{k}^{2}-\Omega_{r}^{2}} t_{0}^{r}, \quad t_{0}^{r}=\left(1+\sum_{k=1}^{N} \frac{c_{k}^{2}}{\left(\omega_{k}^{2}-\Omega_{r}^{2}\right)^{2}}\right)^{-\frac{1}{2}},
$$

It is worth to mention that the matrix $\left\{t_{\mu}^{r}\right\}$ is orthogonal and satisfy the following relations

$$
\sum_{\mu} t_{\mu}^{r} t_{\mu}^{s}=\delta_{r s}, \text { and } \sum_{r} t_{\mu}^{r} t_{\nu}^{r}=\delta_{\mu \nu} .
$$

The Hamiltonian (13) can be rewritten in collective coordinates, which simply reduce to

$$
H=\frac{1}{2}\left(p_{-}^{2}+\omega_{0}^{2} q_{-}^{2}\right)+\frac{1}{2} \sum_{r}\left(P_{r}^{2}+\Omega_{r}^{2} Q_{r}^{2}\right),
$$

where the normal frequencies $\Omega_{r}$ are the solutions of the equation

$$
\omega_{0}^{2}-\Omega_{r}^{2}=\sum_{k=1}^{N} \frac{c_{k}^{2} \Omega_{r}^{2}}{\omega_{k}^{2}\left(\omega_{k}^{2}-\Omega_{r}^{2}\right)} .
$$

Now, we are ready to write the eigenfunctions and energy eigenvalues of the system. The eigenfunctions are given by

$$
\phi_{n_{-}, n_{0}, n_{1}, . .}\left(q_{-}, Q\right)=\phi_{n_{-}}\left(q_{-}\right) \prod_{r=0} \phi_{n_{r}}\left(Q_{r}\right)
$$

where $\phi_{n_{-}}\left(q_{-}\right), \phi_{n_{r}}\left(Q_{r}\right)$ are one dimensional harmonic oscillator eigenfunctions of frequencies $\omega_{0}$ and $\Omega_{r}$ respectively. Whereas the corresponding energy eigenvalues are,

$$
E_{n_{-}, n_{0}, n_{1}, \ldots}=\left(n_{-}+\frac{1}{2}\right) \omega_{0}+\sum_{r=0}\left(n_{r}+\frac{1}{2}\right) \Omega_{r},
$$

where $n_{-}, n_{r}=\{0,1,2, \ldots\}$.

\section{A. Dressed coordinates and states}

In what follows, we introduce the dressed coordinates $q_{A}^{\prime}, q_{B}^{\prime}$ and $q_{k}^{\prime}$, for the atoms $A, B$ and field modes respectively. In terms of this coordinates, we define the dressed states as follows

$$
\psi_{n_{A}, n_{B}, n_{k}}\left(q^{\prime}\right)=\psi_{n_{A}}\left(q_{A}^{\prime}\right) \psi_{n_{B}}\left(q_{B}^{\prime}\right) \prod_{k=1} \psi_{n_{k}}\left(q_{k}^{\prime}\right),
$$

where $\psi_{n_{A}}\left(q_{A}^{\prime}\right), \psi_{n_{B}}\left(q_{B}^{\prime}\right)$ and $\psi_{n_{k}}\left(q_{k}^{\prime}\right)$ are one dimensional harmonic oscillators eigenfunctions, with frequencies $\omega_{A}=\omega_{0}, \omega_{B}=\omega_{0}$ and $\omega_{k}$, respectively. We define such dressed states as the physically realizable states. The dressed state as given by Eq. (21) represent the state in which the atom $A$ is in the energy level $n_{A}$, the atom $B$ is in the energy level $n_{B}$ and there are $n_{k}$ field quanta of frequencies $\omega_{k}$. In general, the dressed states defined above are unstable states, because interaction, such states could decay into other measurable states. For example, the state $\psi_{1,0,0, \ldots, 0}\left(q^{\prime}\right)$ in which the atom $A$ is in its first excited level could decay to its ground state by emission of field quanta or due to the absortion of energy by the atom $B$. In this case, the final possible states are the dressed ones $\psi_{0,0, . .0,1_{k}, 0, . .0}\left(q^{\prime}\right)$ or $\psi_{0,1,0, \ldots, 0}\left(q^{\prime}\right)$.

On the other hand, the dresed ground state $\psi_{0,0,0 \ldots}\left(q^{\prime}\right)$ that describes the two atoms in the ground state and no field quanta, must be stable, according experimental facts. The last one allow us to give an analytical expression for the dressed coordinates $q_{A}^{\prime}, q_{B}^{\prime}$ and $q_{k}^{\prime}$ in terms of collective coordinates or in terms of the bare ones. The stability requires that the dressed ground state $\psi_{0,0, \ldots, 0}\left(q^{\prime}\right)$ must be one of the energy eigenfunctions of the Hamiltonian (17), and it is natural to associate it with 
the ground state of the system, $\phi_{0,0, \ldots, 0}\left(q_{-}, Q\right)$. Thus, we can obtain the dressed coordinates from

$$
\psi_{0,0, \ldots, 0}\left(q^{\prime}\right) \propto \phi_{0,0, \ldots, 0}\left(q_{-}, Q\right) .
$$

The ground state of the system and the dressed ground state are given respectively by

$$
\phi_{0,0, \ldots, 0}\left(q_{-}, Q\right) \propto \mathrm{e}^{-\frac{1}{2}\left(\omega_{0} q_{-}^{2}+\sum_{r} \Omega_{r} Q_{r}^{2}\right)} .
$$

and

$$
\psi_{0,0, \ldots, 0}\left(q^{\prime}\right) \propto \mathrm{e}^{-\frac{1}{2}\left(\omega_{0} q_{A}^{\prime 2}+\omega_{0} q_{B}^{\prime 2}+\sum_{k=1} \omega_{k} q_{k}^{2}\right)} .
$$

In order to find the relation between dressed and collective coordinates, let us define the dressed relative coordinate $q_{-}^{\prime}$ and dressed center of mass coordinate $q_{0}^{\prime}$, in the same way as for the bare ones (12), we have

$$
q_{-}^{\prime}=\frac{1}{\sqrt{2}}\left(q_{A}^{\prime}-q_{B}^{\prime}\right), \quad q_{0}^{\prime}=\frac{1}{\sqrt{2}}\left(q_{A}^{\prime}+q_{B}^{\prime}\right) .
$$

Substituting in terms of relative and center of mass dressed coordinates, the dressed ground state (24), becomes,

$$
\psi_{0,0, \ldots, 0}\left(q^{\prime}\right) \propto \mathrm{e}^{-\frac{1}{2}\left(\omega_{0}{q_{-}^{\prime}}^{2}+\omega_{0}{q_{0}^{\prime}}^{2}+\sum_{k=1} \omega_{k} q_{k}^{\prime 2}\right)} .
$$

Using Eqs. (23) and (26) in (22) we have,

$$
q_{-}^{\prime}=q_{-}, \quad q_{\mu}^{\prime}=\sum_{r=0} \sqrt{\frac{\Omega_{r}}{\omega_{\mu}}} t_{\mu}^{r} Q_{r}, \quad \mu=0, k
$$

where we have considered the property given by Eq. (16). Finally, using Eq. (25) in (27), we are able to write the dressed coordinates for the two atoms and field modes,

$$
\begin{aligned}
q_{A}^{\prime} & =\frac{1}{\sqrt{2}}\left(\sum_{r=0} \sqrt{\frac{\Omega_{r}}{\omega_{0}}} t_{0}^{r} Q_{r}+q_{-}\right), \\
q_{B}^{\prime} & =\frac{1}{\sqrt{2}}\left(\sum_{r=0} \sqrt{\frac{\Omega_{r}}{\omega_{0}}} t_{0}^{r} Q_{r}-q_{-}\right), \\
q_{k}^{\prime} & =\sum_{r=0} \sqrt{\frac{\Omega_{r}}{\omega_{k}}} t_{k}^{r} Q_{r} .
\end{aligned}
$$

As will be shown in the following section, the above relationships will be of fundamental value to perform quantum mechanical calculations.

\section{PROBABILITY AMPLITUDES}

In this section, we obtain the different probability amplitudes between the dressed states introduced in the last section. These quantities will give us the probability of transitions between the different atom-atom-field states. Suppose that at time $t=0$, the state of the system is given by $\psi_{n_{A}, n_{B}, n_{1}, \ldots}\left(q^{\prime}\right)$, where the atom $A$ is in the energy level $n_{A}$, the atom $B$ is in the energy level $n_{B}$ and there are $n_{k}$ field quanta of frequencies $\omega_{k}, k=\{1,2, \ldots\}$. We want to obtain the probability amplitude to find the system at time $t$ in the state $\psi_{m_{A}, m_{B}, m_{1}, \ldots}\left(q^{\prime}\right)$, i.e, the state in which the atom $A$ is in the energy level $m_{A}$, the atom $B$ is in the energy level $m_{B}$, and there are $m_{k}$ field quanta of frequencies $\omega_{k}$. Denoting such probability

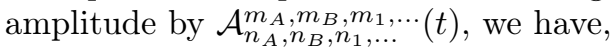

$$
\mathcal{A}_{n_{A}, n_{B}, n_{1}, \ldots}^{m_{A}, m_{B}, m_{1}, \ldots}(t)={ }_{d}\left\langle m_{A}, m_{B}, m_{1}, \ldots\left|\mathrm{e}^{-i \hat{H} t}\right| n_{A}, n_{B}, n_{1}, \ldots\right\rangle_{d},
$$

where the dressed kets $\left|n_{A}, n_{B}, n_{1}, \ldots\right\rangle_{d}$ are such that, $\psi_{n_{A}, n_{B}, n_{1}, \ldots}\left(q^{\prime}\right)=\left\langle q_{A}^{\prime}, q_{B}^{\prime}, q_{1}^{\prime}, \ldots \mid n_{A}, n_{B}, n_{1}, \ldots\right\rangle_{d}$.

For further use, we will focus only on the probability amplitudes between low-level energy atomic states. Let us consider, for example, that at time $t=0$ the atom $A$ is in the first excited level, atom $B$ is on the ground state and there are no field quanta. In this case the state of the system is given by

$$
\psi_{1,0,0, \ldots, 0}\left(q^{\prime}\right)=\psi_{0,0,0, . .}\left(q^{\prime}\right) H_{1}\left(\sqrt{\omega_{0}} q_{A}^{\prime}\right)
$$

Rewriting the above eigenfunction, in terms of collective coordinates, using $\psi_{0,0, \ldots, 0}\left(q^{\prime}\right)=\phi_{0,0, \ldots, 0}\left(q_{-}, Q\right)$, Eqs. (28) and (19), we have

$$
\psi_{1,0,0, \ldots, 0}\left(q^{\prime}\right)=\frac{1}{\sqrt{2}}\left(\phi_{1,0,0, \ldots, 0}+\sum_{r=0} t_{0}^{r} \phi_{0, \ldots, 0,1_{r}, 0, . ., 0}\right) .
$$

At time $t$, this state will evolve to,

$$
\begin{aligned}
\psi_{1,0,0, \ldots, 0}\left(q^{\prime}, t\right)= & \frac{\mathrm{e}^{-i E_{0} t}}{\sqrt{2}}\left(\mathrm{e}^{-i \omega_{0} t} \phi_{1,0,0, . ., 0}+\right. \\
& \left.+\sum_{r=0} t_{0}^{r} \mathrm{e}^{-i \Omega_{r} t} \phi_{0, . ., 0,1_{r}, 0, . ., 0}\right),
\end{aligned}
$$

with $E_{0}=\left(\omega_{0}+\sum_{r} \Omega_{r}\right) / 2$, the ground state energy of the total system.

Now, we can easily calculate the different probability amplitudes associated with the different possible transitions. We can perform the probability amplitudes with these different possibilities as fallows. The probability amplitude to find the state $\psi_{1,0,0, \ldots, 0}$, to remain in the initial state, is obtained by

$$
\begin{aligned}
\mathcal{A}_{1,0,0, \ldots}^{1,0,0, \ldots}(t) & =\int d Q d q_{-} \psi_{1,0,0, \ldots, 0}^{*}\left(q^{\prime}\right) \psi_{1,0,0 \ldots, 0}\left(q^{\prime}, t\right) \\
& =\frac{\mathrm{e}^{-i E_{0} t}}{2}\left(\mathrm{e}^{-i \omega_{0} t}+f_{00}(t)\right)
\end{aligned}
$$

where,

$$
f_{00}(t)=\sum_{r=0}\left(t_{0}^{r}\right)^{2} \mathrm{e}^{-i \Omega_{r} t}
$$

In order to compute the probability amplitude that the atom $A$ decays into its ground state and the atom $B$ jump from its ground state to its first excited level, we 
write such final possible state, $\psi_{0,1,0, \ldots, 0}\left(q^{\prime}\right)$, in terms of collective coordinates,

$$
\psi_{0,1,0, \ldots, 0}\left(q^{\prime}\right)=\frac{1}{\sqrt{2}}\left(\sum_{r=0} t_{0}^{r} \phi_{0, . ., 0,1_{r}, 0, \ldots, 0}-\phi_{1,0,0, . ., 0}\right)
$$

and taking the scalar product with (34) we have,

$$
\mathcal{A}_{1,0,0, \ldots}^{0,1,0, \ldots}(t)=\frac{\mathrm{e}^{-i E_{0} t}}{2}\left(f_{00}(t)-\mathrm{e}^{-i \omega_{0} t}\right) .
$$

In a similar way, considering $\psi_{0, \ldots, 0,1_{k}, 0, \ldots, 0}\left(q^{\prime}\right)$, the state in which the two atoms are in the ground state and there is a field quanta of frequency $\omega_{k}$, could be expressed it in terms of collective coordinates as follow,

$$
\psi_{0, \ldots, 0,1_{k}, 0, \ldots, 0}\left(q^{\prime}\right)=\sum_{r=0} t_{k}^{r} \phi_{0, \ldots, 0,1_{r}, 0 \ldots}
$$

and taking the scalar product with (34) we find the probability amplitude for atom $A$ to decay from its first excited level by emission of a field quanta of frequency $\omega_{k}$,

$$
\mathcal{A}_{1,0,0, \ldots}^{0,0, \ldots, 0,1_{k}, 0, . .}(t)=\frac{\mathrm{e}^{-i E_{0} t}}{\sqrt{2}} f_{0 k}(t)
$$

where

$$
f_{0 k}(t)=\sum_{r=0} t_{0}^{r} t_{k}^{r} \mathrm{e}^{-i \Omega_{r} t}
$$

The states $\psi_{1,0,0, \ldots, 0}\left(q^{\prime}\right), \quad \psi_{0,1,0, \ldots, 0}\left(q^{\prime}\right) \quad$ and $\psi_{0, \ldots, 0,1_{k}, 0, \ldots, 0}\left(q^{\prime}\right)$ are the only possible ones in which the initial state $\psi_{1,0,0, \ldots, 0}\left(q^{\prime}\right)$ can be found at time $t$. To show this one, we take the probabilities $\left|\mathcal{A}_{1,0,0, \ldots}^{1,0,0, \ldots}(t)\right|^{2}$, $\left|\mathcal{A}_{1,0,0, \ldots}^{0,1,0, \ldots}(t)\right|^{2}$ and $\left|\mathcal{A}_{1,0,0, \ldots}^{0, \ldots, 0,1_{k}, 0, \ldots}(t)\right|^{2}$, using the eqs. (35), (38), (40) and (16), we find the following relation

$$
\left|\mathcal{A}_{1,0,0, \ldots}^{1,0,0, \ldots}(t)\right|^{2}+\left|\mathcal{A}_{1,0,0, \ldots}^{0,1,0, \ldots}(t)\right|^{2}+\sum_{k=1}\left|\mathcal{A}_{1,0,0, \ldots}^{0, \ldots, 0,1_{k}, 0, \ldots}(t)\right|^{2}=1,
$$

that shows our assertion. Note that in (42), the summation was performed over all possible frequencies since the emitted field quanta could be of any arbitrary frequency.

Now, considering as the initial state $\psi_{0,1,0, \ldots, 0}\left(q^{\prime}\right)$, the state in which the atom $B$ is in its first excited level, while the atom $A$ is in its ground state, and there are no field quanta, we can find, in similar way as above, all the probability amplitudes related to such initial state. Because atoms $A$ and $B$ are identical, the state of the system can be found at time $t$ in one of the states, $\psi_{1,0,0, \ldots, 0}\left(q^{\prime}\right)$, $\psi_{0,1,0, \ldots, 0}\left(q^{\prime}\right)$ or $\psi_{0, . ., 0,1_{k}, 0, \ldots, 0}\left(q^{\prime}\right)$. We find respectively for each one of these possible states,

$$
\begin{aligned}
\mathcal{A}_{0,1,0, \ldots}^{1,0,0, \ldots}(t) & =\mathcal{A}_{1,0,0, \ldots}^{0,1,0 \ldots}(t), \\
\mathcal{A}_{0,1,0, \ldots}^{0,1,0, \ldots}(t) & =\mathcal{A}_{1,0,0, \ldots}^{1,0,0 \ldots}(t), \\
\mathcal{A}_{0,1,0, \ldots}^{0, \ldots, 0,1_{k}, 0 \ldots}(t) & =\mathcal{A}_{1,0,0, \ldots}^{0, \ldots, 0,1_{k}, 0 \ldots}(t) .
\end{aligned}
$$

In order to shorten the notation for the dressed states, $\psi_{1,0,0, \ldots, 0}\left(q^{\prime}\right), \psi_{0,1,0, \ldots, 0}\left(q^{\prime}\right)$ and $\psi_{0,0, . ., 0,1_{k}, 0, \ldots, 0}\left(q^{\prime}\right)$, we can write the dressed states using Dirac notation, respectively by $|1,0,0, \ldots\rangle_{d},|0,1,0, \ldots\rangle_{d}$ and $\left|0,0, \ldots, 0,1_{k}, 0, \ldots\right\rangle_{d}$. Now, using the results obtained above, we can write the dressed states $|1,0,0, \ldots\rangle_{d}$ and $|0,1,0, \ldots\rangle_{d}$, at time $t$, respectively as

$$
\begin{aligned}
|1,0,0, \ldots ; t\rangle_{d}= & \sum_{k=1} \mathcal{A}_{1,0,0, \ldots}^{0, \ldots, 0,1_{k}, 0 \ldots}(t)\left|0,0, \ldots, 0,1_{k}, 0, . .\right\rangle_{d}+ \\
& +\mathcal{A}_{1,0,0, \ldots}^{1,0,0, \ldots}(t)|1,0,0, \ldots\rangle_{d} \\
& +\mathcal{A}_{1,0,0, \ldots}^{0,1,0 \ldots}(t)|0,1,0, \ldots\rangle_{d},
\end{aligned}
$$

and

$$
\begin{aligned}
|0,1,0, \ldots ; t\rangle_{d}= & \sum_{k=1} \mathcal{A}_{0,1,0, \ldots}^{0, \ldots, 0,1_{k}, 0 \ldots}(t)\left|0,0, \ldots, 0,1_{k}, 0, . .\right\rangle_{d} \\
& +\mathcal{A}_{0,1,0, \ldots}^{0,1,0, \ldots}(t)|0,1,0, \ldots\rangle_{d} \\
& +\mathcal{A}_{0,1,0, \ldots}^{1,0,0 \ldots}(t)|1,0,0, \ldots\rangle_{d}
\end{aligned}
$$

In next section, when we consider the dynamics of the entanglement of specific atomic states, we will use the above time dependent dressed states.

From expressions given by Eqs. (35), (38) and (40), we note that to obtain the time evolution of vector states, as given by Eqs. (44) and (45), we have to compute the coefficients $f_{\mu \nu}$, Eqs. (36) and (41). It is a formidable task since before we have to compute the coefficients $t_{\mu}^{r}$ and collective frequencies $\Omega_{r}$, given by Eqs. (15) and (18). For limiting cases of large (infinite) cavity and very small cavity one can obtain analytical results for $f_{00}$ and $f_{0 k}$. Nevertheless, for intermediate cavity size, finding $f_{00}$ and $f_{0 k}$, becomes a cumbersome task. Then, for intermediate cavity size we have resort to numerical computations. For this purpose Eq. (18) is expressed as [19],

$$
\cot \left(R \Omega_{r}\right)=\frac{\Omega_{r}}{\pi g}+\frac{1}{R \Omega_{r}}\left(1-\frac{R \omega_{0}^{2}}{\pi g}\right)
$$

This transcendental equation can be solved numerically for the collective frequencies $\Omega_{r}$ and using the numerical solutions we can find $\left(t_{0}^{r}\right)^{2}$, the term of Eq. (36), which can be recast in the form,

$$
\left(t_{0}^{r}\right)^{2}=\frac{\eta^{2} \Omega_{r}^{2}}{\left(\Omega_{r}^{2}-\omega_{0}\right)^{2}+\frac{\eta^{2}}{2}\left(3 \Omega_{r}^{2}-\omega_{0}^{2}\right)+\pi^{2} g^{2} \Omega_{r}^{2}},
$$

where $\eta=\sqrt{2 g \Delta \omega_{k}}$. Finally, the infinite sum that appears in (36) or (41) can be done numerically, by noting that for large $\Omega_{r}$, the coefficient $\left(t_{0}^{r}\right)^{2}$ approaches zero as $1 /\left(\Omega_{r}\right)^{2}$.

\section{ENTANGLEMENT OF TWO-ATOM SYSTEM DRIVEN BY QUANTUM FIELD}

Once we know the time evolution of vector states, we can construct the time dependent reduced density operator for the two-atoms system considered in last section 
and this will be useful to study the dynamics of the entanglement through the concurrence quantity. Assuming the state of the system, at time $t=0$, is given by an arbitrary linear combination of the states $|1,0,0, \ldots\rangle_{d}$ and $|0,1,0, \ldots\rangle_{d}$,

$$
|\psi\rangle_{d}=\sqrt{\xi}|1,0,0, \ldots\rangle_{d}+\sqrt{1-\xi} \mathrm{e}^{i \phi}|0,1,0, \ldots\rangle_{d},
$$

where in the first state the atom $A$ is in the first excited level and in the second state, the atom $B$ is in the first excited level. Whereas $\xi$ and $\phi$ are constant parameters that fix the initial conditions. Using Eqs. (44), (45), and (43) we have the time dependent state $|\psi, t\rangle_{d}$, given by

$$
\begin{aligned}
|\psi, t\rangle_{d}= & A(t)|1,0,0, \ldots\rangle_{d}+B(t)|0,1,0, \ldots\rangle_{d}+ \\
& +\sum_{k=1} C_{k}(t)\left|0,0, \ldots, 0,1_{k}, 0, \ldots\right\rangle_{d}
\end{aligned}
$$

where

$$
\begin{aligned}
A(t) & =\sqrt{\xi} \mathcal{A}_{1,0,0, \ldots}^{1,0,0, \ldots}(t)+\sqrt{1-\xi} \mathrm{e}^{i \phi} \mathcal{A}_{1,0,0, \ldots}^{0,1,0, \ldots}(t), \\
B(t) & =\sqrt{\xi} \mathcal{A}_{1,0,0, \ldots}^{0,1, \ldots, \ldots}(t)+\sqrt{1-\xi} \mathrm{e}^{i \phi} \mathcal{A}_{1,0,0, \ldots}^{1,0,0, \ldots}(t), \\
C_{k}(t) & =\left(\sqrt{\xi}+\sqrt{1-\xi} \mathrm{e}^{i \phi}\right) \mathcal{A}_{1,0,0, \ldots}^{0,0, \ldots, 0,1_{k}, 0 \ldots} .
\end{aligned}
$$

The density operator for the state (49) is,

$$
\hat{\rho}(t)=|\psi, t\rangle_{d d}\langle\psi, t| .
$$

Tracing out over all field quanta degrees of freedom in (53) we have the reduced density matrix for the two-atom system,

$$
\hat{\rho}_{r}(t)=\sum_{n_{1}, n_{2}, \ldots=0}{ }_{d}\left\langle n_{1}, n_{2}, \ldots|\hat{\rho}(t)| n_{1}, n_{2}, \ldots\right\rangle_{d},
$$

where $\left|n_{1}, n_{2}, \ldots\right\rangle_{d}$ are states restricted to the sub-space of the degrees of freedom of the quantum field. Using (49), the reduced density operator (54) becomes,

$$
\begin{aligned}
\hat{\rho}_{r}(t)= & E(t)|0,0\rangle_{d d}\langle 0,0|+ \\
& +A(t) B^{*}(t)|1,0\rangle_{d d}\left\langle 0,1\left|+A^{*}(t) B(t)\right| 0,1\right\rangle_{d d}\langle 1,0|+ \\
& +|A(t)|^{2}|1,0\rangle_{d d}\left\langle 1,\left.0|+| B(t)\right|^{2} \mid 0,1\right\rangle_{d d}\langle 0,1|
\end{aligned}
$$

with $E(t)$ given by

$$
\begin{aligned}
E(t) & =\sum_{k=1}\left|C_{k}(t)\right|^{2} \\
& =1-|A(t)|^{2}-|B(t)|^{2} .
\end{aligned}
$$

where in passing to the second relation, we have used Eqs. (50)-(52) and (42). In the basis $\left\{|0,0\rangle_{d},|1,0\rangle_{d},|0,1\rangle_{d},|1,1\rangle_{d}\right\}$, the reduced density matrix is

$$
\rho_{r}(t)=\left(\begin{array}{cccc}
E(t) & 0 & 0 & 0 \\
0 & |A(t)|^{2} & A(t) B^{*}(t) & 0 \\
0 & A^{*}(t) B(t) & |B(t)|^{2} & 0 \\
0 & 0 & 0 & 0
\end{array}\right) .
$$

To measure the entanglement created by the two-atom system, we use the concurrence as a measure of entanglement, which was introduced by Wootters [34]. The concurrence can be obtained from the reduced density matrix $\rho_{r}$. Considering the spin-flipped state, $\mathbf{R}(t)=$ $\rho_{r}(t) \sigma_{2} \otimes \sigma_{2} \rho_{r}(t)^{*} \sigma_{2} \otimes \sigma_{2}$, and using (57), we have

$$
\mathbf{R}(t)=2\left(\begin{array}{cccc}
0 & 0 & 0 & 0 \\
0 & |A(t)|^{2}|B(t)|^{2} & |A(t)|^{2} A(t) B^{*}(t) & 0 \\
0 & |B(t)|^{2} A^{*}(t) B(t) & |A(t)|^{2}|B(t)|^{2} & 0 \\
0 & 0 & 0 & 0
\end{array}\right) .
$$

Therefore, the concurrence is defined as $\mathcal{C}\left(\rho_{r}\right)=$ $\max \left\{0, \lambda_{1}-\lambda_{2}-\lambda_{3}-\lambda_{4}\right\}$, where the $\lambda_{i}$ 's are the square root of the eigenvalues of matrix (58). After some algebraic manipulation, we obtain

$$
\mathcal{C}(R, g, t, \xi, \phi)=2|A(t) B(t)| .
$$

The concurrence, as given above, depends through $A(t)$ and $B(t)$ of frequency $\omega_{0}$, cavity radius $R$, coupling constant with the scalar field $g$, initial conditions $\xi$ and $\phi$, and certainly depends of time $t$. Some particular results can be obtained, such as taking $\xi=1 / 2$ and $\phi=0$ in Eq. (48). In this case the initial state

$$
|\psi\rangle_{d}=\frac{1}{\sqrt{2}}\left(|1,0,0, \ldots\rangle_{d}+|0,1,0, \ldots\rangle_{d}\right)
$$

is a symmetric maximally entangled state. Using Eqs. (35) and (38) in (50)-(51), in this case the concurrence (59) reduce to the following expression

$$
\mathcal{C}(t, \xi=1 / 2, \phi=0)=\left|f_{00}(t)\right|^{2},
$$

which coincide with the probability of a dressed atom to remain in its first excited level at time $t$ [19].

Another limiting case can be obtained when taking as initial state a maximally antisymmetric state, corresponding to $\xi=1 / 2$ and $\phi=\pi$ in Eq. (48), the concurrence simply becomes

$$
\mathcal{C}(t, \xi=1 / 2, \phi=\pi)=1,
$$

the two-atoms system is maximally entangled through the time, independent of any cavity parameter, frequency $\omega_{0}$ or coupling constant $g$.

On the other hand, if initially we have a disentangled state, for example $\xi=0, \phi=0$ in Eq. (48),

$$
|\psi\rangle_{d}=|0,1,0, \ldots\rangle_{d},
$$

the concurrence becomes

$$
\mathcal{C}(t, \xi=0, \phi=0)=\frac{1}{2}\left|f_{00}(t)^{2}-\mathrm{e}^{-2 i \omega_{0} t}\right| .
$$

For above or other initial conditions we can solve the concurrence either analytically or numerically, depending if cavity radius is infinite or finite. In this sense, in what follows we consider first the infinite cavity radius case. 


\section{A. Concurrence in free space, $R \rightarrow \infty$.}

From Eqs. (59), (50), (51), (35) and (38), we note that the concurrence, for the two-atom state (48) at time $t$, can be expressed through the time dependent coefficient $f_{00}(t)$. For infinite cavity, $R \rightarrow \infty$, the coefficient $f_{00}(t)$ is given by [19], [29],

$$
f_{00}(t)=2 g \int_{0}^{\infty} d x \frac{x^{2} \mathrm{e}^{-i x t}}{\left(x^{2}-\omega_{0}^{2}\right)^{2}+\pi^{2} g^{2} x^{2}} .
$$

Therefore, for sufficiently large $t \rightarrow \infty$, we have $f_{00}(t) \rightarrow$ 0 , thus, the concurrence becomes

$$
\mathcal{C}(t \rightarrow \infty)=\frac{1}{2}-\sqrt{\xi(1-\xi)} \cos \phi .
$$

From above equation we can note that, for any initial state of the type given by (48), in general the twoatom system is never disentangled for large $t$ unless $\sqrt{\xi(1-\xi)} \cos \phi=1 / 2$. It is not difficult to see that above condition is satisfied only if $\xi=0.5$ and $\phi=0$, i.e, the only initial state of the type (48) that becomes disentangled for large time $t$ is the symetric state given by Eq. (60). For this initial state, in Fig. 1 (a), the time evolution of the concurrence is depicted in solid line for $g=\omega_{0}$, where concurrence decays almost exponentially from 1 to 0 . For other initial states, the two-atom system never disentangle, including an initial non entangled state as the one given by Eq. (63). In this case, as showed in Fig. 1 1 b, solid curve, concurrence increases from 0 to $1 / 2$ according (66).

On the other hand, at time $t \rightarrow \infty$ a maximal entangled state is possible if $\sqrt{\xi(1-\xi)} \cos \phi=-1 / 2$, a relation satisfied only if $\xi=1 / 2$ and $\phi=\pi$, i.e, if the initial state is the antisymmetric one for which the system is maximally entangled all the time, Eq. (62).
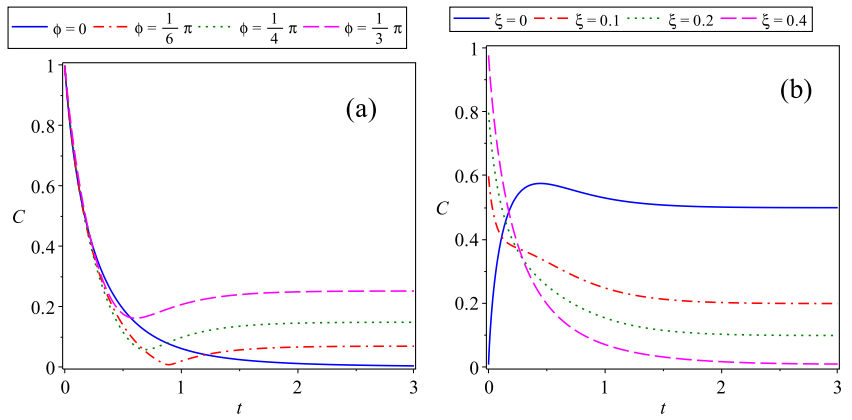

Figure 1. (Color online) (a) The time evolution of the concurrence for fixed $g=\omega_{0}$, assuming initial state (48) $\xi=0.5$, and for a range of values $\phi$. (b) The time evolution of concurrence for $g=\omega_{0}$, assuming initial state (48) $\phi=0$, and for a range of values $\xi$. Time is in units of $\omega_{0}^{-1}$.

For other initial states and finite time, $t$, we show in Fig. 1 the time evolution of the concurrence for coupling constant $g=\omega_{0}$. In Fig. 1 (a) it is considered four initial states with common $\xi=0,5$ and $\phi=0, \pi / 6, \pi / 4, \pi / 3$. In
Fig. 1 1 (b), it is considered four initial states with fixed $\phi=0$ and $\xi=0,0.1,0.2,0.4$. In all these cases we note a non oscillatory behavior of the concurrence as a function of time and in agreement with Eq. (66), the concurrence approaches a definite value at $t \rightarrow \infty$. For other values of the coupling constant $g$ the qualitative behaviour in time will be the same. If $g>\omega_{0}$, the assymptotic value of the concurrence will be reached faster than where $g=\omega_{0}$ and if $g<\omega_{0}$ we will have a contrary effect.

From Fig. 1 (a), we note that for some particular initial initial states, for example $\xi=0.5, \phi=\pi / 6$, the concurrence approaches zero for finite time $t$. It is not difficult to find the condition for a disentangled state at finite time $t$. From Eq. (59), concurrence will be zero if $A(t)=0$ or $B(t)=0$, and using Eqs. (50) and (51) we get

$$
\begin{aligned}
& (2 \xi-1)\left(\left|f_{00}(t)\right|^{2}+1\right)+2 \Re\left(f_{00}(t) \mathrm{e}^{i \omega_{0} t}\right)=0, \\
& e^{i \phi}= \pm \frac{\sqrt{\xi}}{\sqrt{1-\xi}} \frac{\left(f_{00}(t)+\mathrm{e}^{-i \omega_{0} t}\right)}{\left(f_{00}(t)-\mathrm{e}^{-i \omega_{0} t}\right)} .
\end{aligned}
$$

Above equations, valid for arbitrary cavity size, must be used as follows. Given $\xi$ and $\phi$ the concurrence must be zero at time $t$, if and only if the pair of Eqs. (67)- 68 are satisfied simultaneusly. Of course for arbitrary values of $\xi$ and $\phi$ above pair of equations are in general not satisfied for any time $t$. For infinity cavity size the function $f_{00}(t)$ given by Eq. (65) is non oscillating in time, consequently concurrence could vanish only for some finite values of $t$ and for very restricted initial conditions. The situation will be different when we consider in the next subsection a cavity of finite size, where $f_{00}(t)$ is an oscillating function of time. In such a case it will be possible $\mathcal{C}=0$, many times, consequently we will have death and revival in time of entanglement for some initial states.

\section{B. Concurrence for finite cavity radius.}

Solving numerically the Eq. (46), we can find the concurrence using the Eq. (59). Therefore, in what follows, we will illustrate some plots of concurrence for a variety of situations, as a function of time $t$.

In Fig. 2, we illustrate the time evolution of concurrence, assuming fixed parameter cavity radius, $R=\omega_{0}^{-1}$, and initial conditions $\xi=0$ and $\phi=0$, [fully disentangled condition, see Eqs. [63) - [64)], as a function of time $t$, for a range of values of $g=\left\{0.01 \omega_{0}, 0.2 \omega_{0}, 0.5 \omega_{0}, \omega_{0}, 2 \omega_{0}\right\}$ from top to bottom. In the top of Fig. 2 is illustrated the time evolution curve for $g=0.01 \omega_{0}$, and one can observe the curve is some what periodic $\mathcal{C} \approx\left|\cos \left(\omega_{0} t\right)\right|$. In below the top of Fig. 2, is depicted for $g=0.1 \omega_{0}$, thus, the periodicity is definitely wrecked, although the curve still holds some periodic pattern. For the middle $\left(g=0.5 \omega_{0}\right)$ and above the below of Fig. 2, for fixed $g=\omega_{0}$, the periodic curve is strongly modified and becomes almost a random curve without any periodicity. In bottom of Fig. 2] is illustrated the curve for $g=2 \omega_{0}$, and the concurrence fluctuate in time around a constant $\mathcal{C}=0.5$, 


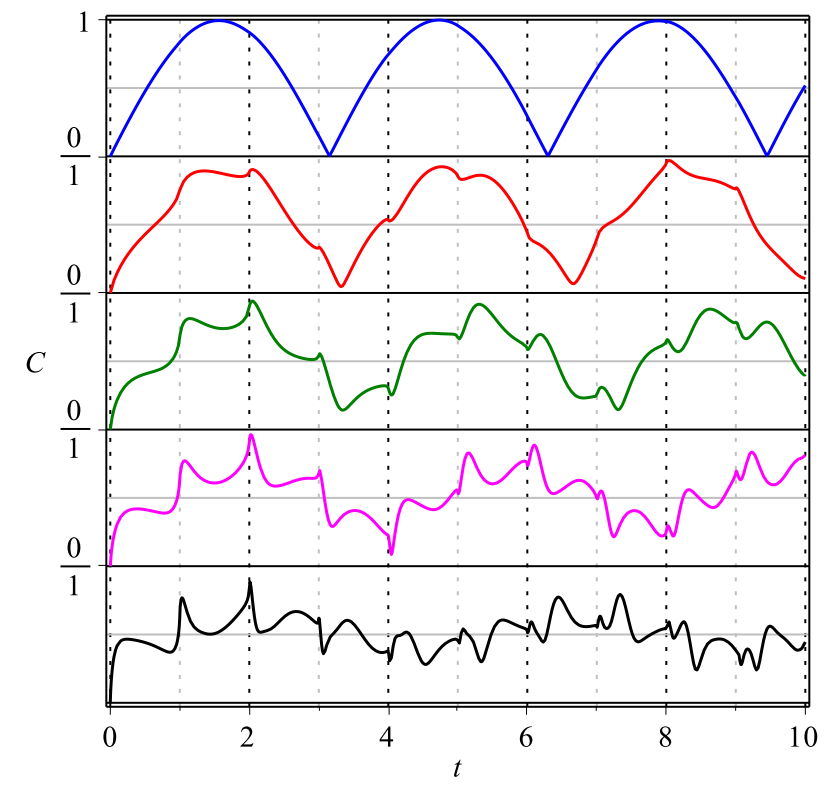

Figure 2. (Color online) The time evolution of the concurrence for an initial state (48) $\xi=0$ and $\phi=0$, assuming fixed $R \omega_{0}=$ 1 , for a range of values of $g=\left\{0.01 \omega_{0}, 0.1 \omega_{0}, 0.5 \omega_{0}, \omega_{0}, 2 \omega_{0}\right\}$, from top to bottom, respectively. Time is in units of $\omega_{0}^{-1}$.

with small short quasi-random oscillations and no periodicity is observed. From these curves, we can conclude that for fixed cavity radius, the concurrence oscilates for suficiently weak coupling constant between its minimum and maximum value, i.e we have a constant revival and death of entanglement when the two atom-system evolves in time. When coupling constant is increased, such effect is suppressed and the concurrece oscillates around its infinity cavity value for large $t$ (compare solid line of Fig. 1.(b) with the curve at bottom of Fig. 2).

In Fig. 3, is illustrated the time evolution of the concurrence in a cavity, for the same initial state 63. above for fixed $g=\omega_{0}$, but now for variable cavity radius $R$. (a) Displays for fixed $R=0.01 \omega_{0}^{-1}$, top curve shows the time evolution from $t=0 \ldots 10$ (in units of $\omega_{0}^{-1}$ ), below top curve shows after elapsed a long time, for an interval $t=10^{2} \ldots 10^{2}+10$, while the bottom curve shows after elapsed a too long time, for an interval $t=10^{9} \ldots 10^{9}+10$. We can observe, the concurrence for small cavity behaves almost periodically, given in the limiting case by $\mathcal{C} \approx\left|\cos \left(\omega_{0} t\right)\right|$ this relation is still valid even after elapsed a too long time. (b) Displays for fixed $R=0.1 \omega_{0}^{-1}$, following the same criterion as in Fig. 3 (a), $t=0 . .10$ (top), $t=10^{2} . .10^{2}+10$ (below top) and $t=10^{9} . .10^{9}+10$ (bottom). Here, we observe the rising of a high-frequency oscillation with small amplitude, but the low frequency oscillation still follows a pattern of periodicity $\mathcal{C} \approx\left|\cos \left(\omega_{0} t\right)\right|$. In Fig. 3-(c) is illustrated similar to Figs. 3 (a) and 3f(b) for $R=0.5 \omega_{0}^{-1}$. In this case, the high frequency oscillation is of order of $\omega_{0}$, that means the combination gives quasi-random oscilla-
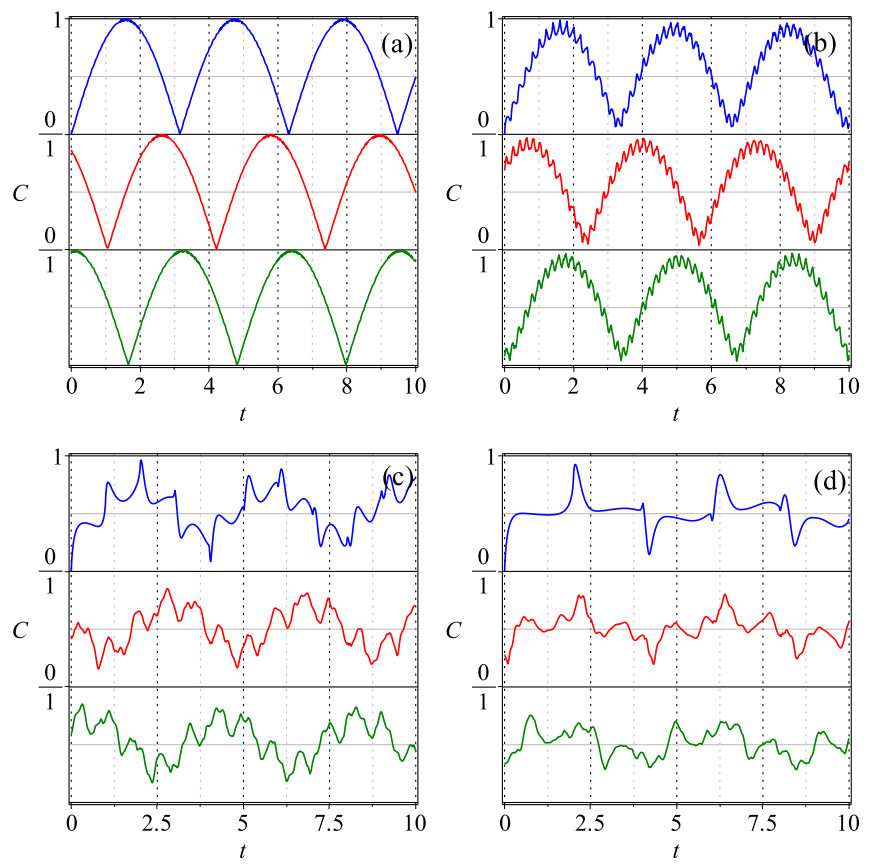

Figure 3. (Color online) Concurrence as a function of time, for an initial state $\xi=0$ and $\phi=0$, and considering fixed $g=\omega_{0}$. (a) Displays for fixed $R \omega_{0}=0.01$, top curves shows the time evolution from $t=0 \ldots 10$, below top curve shows after elapsed a long time, for an interval $t=10^{2} \ldots 10^{2}+10$, while bottom curve shows after elapsed a too long time, for an interval $t=10^{9} \ldots 10^{9}+10$. (b) Displays for fixed $R \omega_{0}=0.1$, following same criterion as in (a), for $t=0 . .10$ (top), for $t=$ $10^{2} . .10^{2}+10$ (below top) and for $t=10^{9} . .10^{9}+10$ (bottom). (c) Similarly to (a) and (b) illustrates for $R \omega_{0}=0.5$. (d) For $R \omega_{0}=1$. Time is in units of $\omega_{0}^{-1}$.

tion. For long time one can observe the low frequency oscillation still remains a pattern of oscillation. We can also show the behavior of this curve after elapsed a too long time, and still remains a similar curve compared for small $t$. Finally, in Fig. 3. (d), is illustrated for $R=\omega_{0}^{-1}$, a cavity large enough compared to the case of Fig. 3 (a). In this panel we can observe the low energy frequency oscillation is vanished and high-frequency oscillation was also wrecked, after elapsed a short time there is only some well pronounced peak at $t=2 R$ (the time necessary for field quanta to go and come back from the center to the spherical cavity wall), but as soon as the time evolves these peaks vanishes and after elapsed a too long time disappear definitely. From the curves of Fig. 3 , we conclude that for sufficiently small cavity radius, the concurrence oscillates between its minimum $\mathcal{C}=0$ and maximum value $\mathcal{C}=1$, consequelty we will have again a an almost periodical revival and death of entanglement for two-atom states. As for large coupling constant, this behaviour will be suppressed for suficiently large cavity radius, where concurrence will oscillate around $\mathcal{C}=0.5$, according to its $R \rightarrow \infty$ assymptotic value, Eq. (66).

Considering as initial state the maximally symmetric 

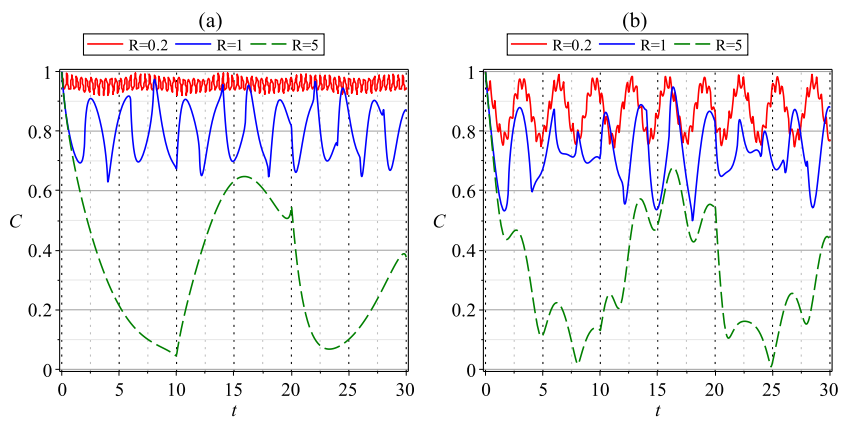

Figure 4. (Color online) (a) Concurrence as a function of time, for an initial state $\xi=0.5$ and $\phi=0$, and considering fixed $g=\omega_{0}$, and for $R \omega_{0}=0.2, R \omega_{0}=1$ and $R \omega_{0}=5$. (b) Concurrence as a function of time, for an initial state $\xi=0.5$ and $\phi=\frac{\pi}{5}$, for the same parameters of (a). Time and cavity radius are given in units of $\omega_{0}^{-1}$.

state (60), $, \xi=0.5, \phi=0$ it is illustrated in Fig. 44(a) the time evolution of the concurrence, assuming fixed $g=\omega_{0}$. We considerer $R=0.2 \omega_{0}^{-1}$ (red/gray solid curve), $R=\omega_{0}^{-1}$ (blue/black solid line) and $R=5 \omega_{0}^{-1}$. We note that for suficiently small cavity radius the concurrence oscillates below its maximum value 1 never decreasing appreciably from this value. In this case the initial maximally entangled state will remain almost maximally entangled all the time. Note that this behaviour is dramatically different from the free space case, $R \rightarrow \infty$, where the initial symmetric state is the only one that becomes disentangled for sufficiently large time. According results above, we expect the same behaviour for sufficiently small coupling constant. When the cavity radius is increased, the minimum for concurrence decreases, and the frequency of oscillation in time increases with $R$. Agai, we expect the same behaviour for increasing coupling constant $g$. Finally in Fig. 4 (b), is depicted the time evolution for the initial state $\xi=0.5, \phi=\pi / 6$,

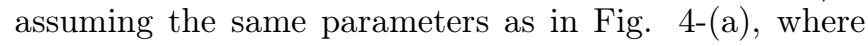
we observe qualitativelly the same behaviour as in above case.

\section{CONCLUSIONS}

In this work, we considered the study of the entanglement of a two-atom system driven by the vacuum quantum field inside a spherical cavity, using the dressed coordinates approach. The time evolution entanglement was analyzed, for a variety of initial states composed as a superposition of atomic states, Eq. (48). For free space, $R \rightarrow \infty$, we find that concurrence approaches assymptotically a fixed value at sufficiently large time. From this result we concluded that with exception of the initial symmetric maximally entangled state, $\xi=0.5, \phi=0$ all the other states become or remain entangled for sufficiently large time. For the initial state $\xi=0.5, \phi=0$ the concurrence decreases, as a function of time, almost exponentially from its maximum value to zero. Also we showed that there are very restricted initial states, who satisfies Eqs. (67)-(68), for which the two-atom state becomes untangled for a finite value of time. Anyway, for large $t$ also those states become or remain entangled.

For finite cavity radius, we found that concurrence in general behaves almost as a periodic function of time, for sufficiently small cavity radius or coupling constant. For the initial disentangled state $\xi=0, \phi=0$, the concurrence oscilates almost periodically for small cavity radius or small coupling constant, between $\mathcal{C}=0$ and $\mathcal{C}=1$. When $R$ or $g$ are increased the aforementioned oscillation is supressed and concurrence approaches its infinity cavity size value, $\mathcal{C}=1 / 2$, see Figs. 2 and 3 On the other hand for the initial symmetric maximally entangled state, $\xi=0.5, \phi=0$, the concurrence is always close to $\mathcal{C}=1$, when the cavity radius or the coupling constant are sufficiently small and for larger cavity radius or coupling constant, the concurrence decays considerably from its initial value, leading to a small concurrence at $t=2 R$, see Fig. 田(a).

In general, when the system is initially maximally entangled (disentangled) after a time, proportional to the cavity radius, had elapsed the system becomes again strongly entangled (disentangled), particularly during the first oscillations, later this phenomenon could be wrecked depending on the initial condition. Another interesting result we found is the behavior of the concurrence after too long time in the future, for large $(R \neq \infty)$ cavity size, apparently there is no pattern resembling some periodicity.

As pointed out earlier, for fre space, $R \rightarrow \infty$, there are some initial entangled states that become disentangled for finite values of time that satisfy Eqs. (67)- (68). As showed such condition is valid for any cavity radius and since for finite cavity radius, $f_{00}(t)$ has an almost periodic behavior for suficiently small cavity or coupling constant, then we expect and oscillatory behaviour for concurrence between its initial value and $\mathcal{C}=0$, i.e, we will have sudden death and revival for those initial conditions, according to the one found in Ref. [32], at zero temperature. We have to remark here that althought the hamiltonian given by Eq. (1) is the same used in Ref. [32], physically the system we treat here is very different, since if written in terms of dressed coordinates (that we take as the physical coordinates) hamiltonian (1) will be very different to the one of Ref. [32].

Also, independent of the cavity radius or other parameters, we found that the initial anti-symmetric maximally entangled state, corresponding to $\xi=0.5, \phi=\pi$, remains fully entangled all the time. The reason for this behavior is because such initial state is an eigenfunction of the total system and consequently remains stable. At this point we have to mention that a similar result has been found recently in Ref. [35], where the authors showed at first order in perturbation theory, that an initial antisymmetric two-atom state remains stable when the spatial separation between the atoms is zero. 
We call atention to the fact that in Ref. 36] the authors considered the same model here. However the authors considered the time evolution of a superposition of dressed states related to the center of mass and relative coordinates. Here we have defined, dressed coordinates and states for the atoms $A$ and $B$, consequently our results and conclusions are quit different.

In this work we considered the case in which the field is initially in the vacuum state and this situation is physically equivalent to one in which the field system is at zero temperature. Then, all above conclusions are valid in such situation and our results are similar to the found in Ref. [32], for $T=0$ with one exception below. We have found in general two phases, suden death and revival (SDR) and non suden death (NSD) depending on the ini- tial state, cavity radius and coupling constant. But when considering as initial state the symmetric maximally entangled one we found sudden death (SD), at large time in free space, $R \rightarrow \infty$. A natural extension of this work is to consider finite temperature effects, where the dynamical entanglement could show a SD phase for another initial states and perhaps for finite cavity size. We expect to report about that elsewhere.

\section{ACKNOWLEDGMENTS}

This work was partially supported by Brazilian agencies CNPq and FAPEMIG.
[1] M.A. Nielsen, I.L. Chuang, Quantum Computation and Quantum Information; Cambridge University Press: Cambridge, UK, (2000).

[2] C.H. Bennett, G. Brassard, C. Crépeau, R. Jozsa, A. Peres, and W. K. Wootters, Phys. Rev. Lett. 70, 1895 (1993).

[3] D. Bouwmeester, J. W. Pan, K. Mattle, M. Eibl, H. Weinfurter, A. Zeilinger, Nature 390, 575 (1997).

[4] U. Marzolino, A. Buchleitner, Phys. Rev. A 91, 032316 (2015).

[5] C. H. Bennett, D. P. DiVincenzo, Nature 404, 247 (2000).

[6] F. G. Deng, G. L. Long, X. S. Liu, Phys. Rev. A 68, 042317 (2003).

[7] R.G. DeVoe and R.G. Brewer, Phys. Rev. Lett. 762049 (1996).

[8] Q.A. Turchette, C.S. Wood, B.E. King, C.J. Myatt, D. Leibfried, W.M. Itano, C. Monroe, and D.J. Wineland, Phys. Rev. Lett. 81, 3631 (1998).

[9] E. Hagley, X. Maitre, G. Nogues, C. Wunderlich, M. Brune, J. M. Raimond, and S. Haroche, Phys. Rev. Lett. 79, 1 (1997).

[10] S. Schneider and G.J. Milburn, Phys. Rev. A 65, 042107 (2002).

[11] M.S. Kim, J. Lee, D. Ahn, and P.L. Knight, Phys. Rev. A 65, 040101(R) (2002).

[12] M.B. Plenio, S.F. Huelga, A. Beige, and P.L. Knight, Phys. Rev. A 59, 2468 (1999).

[13] A. Beige, S. Bose, D. Braun, S.F. Huelga, P.L. Knight, M.B. Plenio, and V. Verdal, J. Mod. Opt. 47, 2583 (2000).

[14] C. Cabrillo, J.I. Cirac, P. Garcia-Fernandez, and P. Zoller, Phys. Rev. A 59, 1025 (1999).

[15] R.H. Dicke, Phys. Rev. 93, 99 (1954).

[16] Z. Ficek and R. Tanas, J. Mod. Opt. 50, 2765 (2003)

[17] R. Tanas and Z. Ficek, J. Opt. B6, s90 (2004).

[18] R. Tanas and Z. Ficek, Fortschr. Phys. 51, 230 (2003)
[19] N. P. Andion, A.P.C. Malbouisson and A. Mattos Neto, J.Phys. A34, 3735 (2001).

[20] G. Flores-Hidalgo, A.P.C. Malbouisson and Y.W. Milla, Phys. Rev. A, 65, 063414 (2002), arXiv:physics/0111042

[21] G. Flores-Hidalgo and A.P.C. Malbouisson, Phys. Rev. A66, 042118 (2002), arXiv:quant-ph/0205042.

[22] R. G. Hulet, E. S. Hilfer, D. Kleppner, Phys. Rev. Lett. 55, 2137 (1985).

[23] W. Jhe, A. Anderson, E. A. Hinds, D. Meschede, L. Moi and S. Haroche, Phys. Rev. Lett. 58, 666 (1987).

[24] G. Flores-Hidalgo and A. P. C. Malbouisson, Phys. Lett. A311, 82 (2003), arXiv:physics/0211123.

[25] G. Flores-Hidalgo and Y. W. Milla, J. Phys. A: Math. Gen. 38, 7527 (2005), arXiv:physics/0410238

[26] R. Casana, G. Flores-Hidalgo and B. M. Pimentel, Phys. Lett. A337, 1 (2005), arXiv:physics/0410063

[27] G. Flores-Hidalgo and A. P. C. Malbouisson, Phys. Lett. A337, 37 (2005), arXiv:physics/0312003.

[28] R. Casana, G. Flores-Hidalgo and B. M. Pimentel, Physica A374, 600 (2007), arXiv: physics/0506223.

[29] G. Flores-Hidalgo, J. Phys. A: Math. Gen. 40, 13217 (2007).

[30] U. Weiss, Quantum Dissipative Systems, (World Scientific Publishing Company; 3 edition, 2008).

[31] C.-H. Chou, T. Yu, and B. L. Hu, Phys. Rev. E 77, 011112 (2008).

[32] J. P. Paz and A. J. Roncaglia, Phys. Rev. Lett. 100, 220401 (2008).

[33] H. R. Wei, F. G. Deng, Phys. Rev. A 88, 042323 (2013).

[34] W.K. Wootters, Phys. Rev. Lett. 80, 2245 (1998); S. Hill and W.K. Wootters, Phys. Rev. Lett. 78, 5022 (1997).

[35] E. Arias, J. G. Dueñas, G. Menezes and N. F. Svaiter, Boundary effects on radiative processes of two entangled atoms, arXiv:1510.00047.

[36] E. G. Figueiredo, C. A. Linhares, A. P. C. Malbouisson and J. M. C. Malbouisson, Phys. Rev. A 84 045802, (2011). 